\title{
Design and Simulation of a Sun Tracking Solar Power System
}

\section{Dr. Liping Guo, Northern Illinois University}

Dr. Liping Guo received his B.E. in Automatic Control from the Beijing Institute of Technology at Beijing, China in 1997. She earned her M.S. and Ph.D. degrees in Electrical and Computer Engineering from Auburn University in 2001 and 2006, respectively. She is currently an assistant professor in the Electrical Engineering Technology program in the Technology Department at the Northern Illinois University. Her research and teaching interests are in the areas of power electronics, renewable energy, embedded systems and automatic control. Dr. Guo is a senior member of the IEEE and a member of the ASEE.

\section{Mr. Jingbo Han, Northern Illinois University}

Jingbo Han earned the M.S in Electrical Engineering from Northern Illinois University in 2010.

\section{Dr. Andrew W Otieno, Associate Professor}

Dr. Andrew W. Otieno is an associate professor in the Department of Technology at Northern Illinois University. He holds a Ph.D. in Mechanical Engineering from Leeds University in the United Kingdom. Dr. Otieno has worked in various capacities at several institutions both in the United States and abroad. Since joining the Department of Technology, Dr. Otieno has actively participated in curriculum development. He has extensively revised and developed new labs in the area of manufacturing engineering, including an automation laboratory in the department. His research interests include structural health monitoring, finite element modeling, machining processes, eco-machining, machine vision applications in tool wear monitoring and micro-machining processes. He has experience in hardware/software interfacing with special applications in machine vision and PLC. He is a member of the ASEE, ASME, EWB and SME. Dr. Otieno has published several articles in the areas of machine vision applications, structural health monitoring, eco-machining, modeling of machining processes and manufacturing education. 


\title{
Design and Simulation of a Sun Tracking Solar Power System
}

\begin{abstract}
Global energy consumption is dramatically increasing due to higher standard of living and the increasing world population. The world has limited fossil and oil resources. As a consequence, the need for renewable energy sources becomes more urgent. With the fast development of renewable energy technology, it proposes increasing demand for the higher education. This project is funded by the National Science Foundation Transforming Undergraduate Education in STEM (TUES) program from May 2012 to April 2015. As part of the objectives of the project, a sun tracking solar power system will be designed and developed as a teaching tool for the laboratory.

This paper describes the design and simulation of a sun tracking solar power system. The simulation is realized on Matlab/Simulink platform. The simulation consists of four modules: solar tracking cells, signal conditioning circuit, controller, and motor. The simulation provides an excellent platform for undergraduate engineering technology students to study the design and theory of a sun tracking solar power system.
\end{abstract}

\section{Introduction}

The efficiency of the photovoltaic (PV) system depends on the climate conditions of solar radiation, ambient temperature and wind speed, matching of the system with the load and appropriate placement of the solar panels. A majority of solar panels in use today are stationary and therefore do not consistently output the maximum amount of power that they can actually produce. A solar tracker will track the sun throughout the day and adjust the angle of the solar panel to make the sun normal to the solar panels at all times. The orientation of the solar panels may increase the efficiency of the conversion system from $20 \%$ up to 50\%. [1-3]. The sun tracking solar power system is a mechatronic system that integrates electrical and mechanical systems, and computer hardware and software.

This paper describes the Matlab/Simulink simulation of a sun tracking solar power system. The simulation will be used for demonstration and experiments to help the students study theory of the system. The solar tracker follows the sun from east to west during the day. More energy is collected by controlling the solar panel to follow the sun like a sunflower. After simulation is complete, a physical system will be implemented.

\section{Design of a Sun Tracking Solar Power System}

The main components in the solar tracking system are standard photovoltaic solar panels (PV), a deep cycle rechargeable battery, battery charge controller, microcontroller, signal conditioning circuits, motor, and motor drive. The block diagram of the system is shown in Figure 1. Three solar panels are used. One main solar panel powers the system, and two low voltage tracking cells to obtain information on the sun angle. The main solar panel provides all the power for the system and charges the battery. The other two small tracking cells work as angle detectors. They are mounted on two 45 degree wedges to detect the exact angle in which the main solar panel 
must face to gain maximum power output. When the sun is perfectly normal, the voltages on both cells are equal, and solar panel stays in its position. However, if the main solar panel is not normal to the sun, the sun angles on the two small solar panels are different [4-6]. The microcontroller gives an output to the motor based on the difference of voltages to rotate the main solar panel to be normal to the sun.

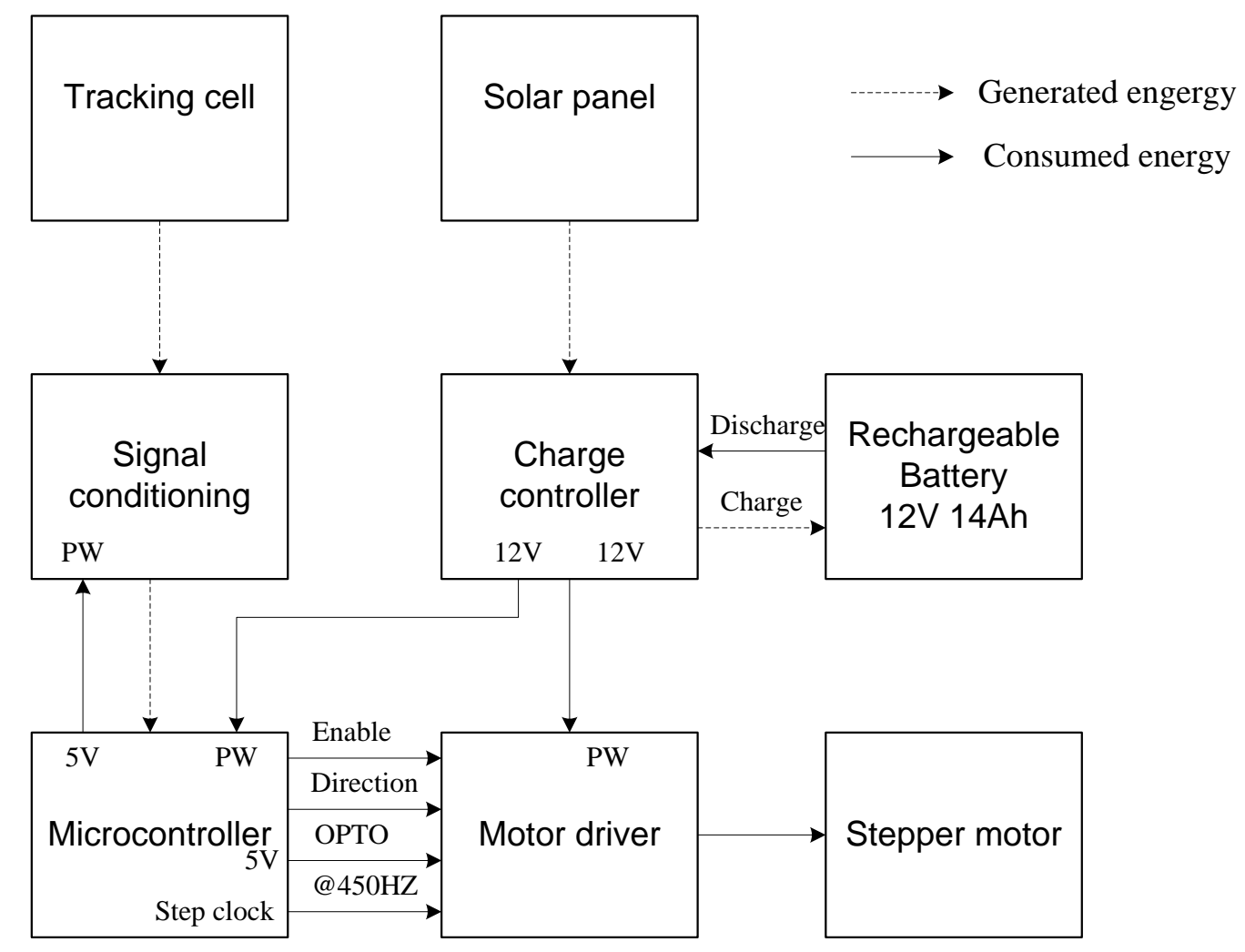

Figure 1. System Block Diagram

\section{Simulation of a Sun Tracking Solar Power System}

The simulation is realized on Matlab/Simulink platform. The simulation consists of four modules: tracking cells, signal conditioning circuit, controller, and motor. The PV tracking cells detect light intensity and convert it into current. The PV tracking cells work as angle detectors. They are mounted on two 45 degree wedges to detect the exact angle in which the main solar panel must face to gain maximum power output. The current is amplified using the signal conditioning circuit, and sent to the microcontroller. The microcontroller uses different control algorithms to generate a signal to control the motor to rotate the main solar panel perpendicular to the sun. An embedded Matlab function simulate the control algorithm and mathematically generate PWM signal to drive the motor. Finally, the motor module consists of a stepper motor and motor drive. The motor module generates mechanical movement of rotation in terms of angle. The simulation provides an excellent platform for undergraduate engineering technology students to study a sun tracking solar power system. 


\section{Solar Tracking Cell Module}

A solar tracking cell generates current when incident light falls on its surface. The amount of current generated is proportional to the light and is determined by the flux density. In Simulink, the model of solar cell can be found in the library of Sources in SimElectronics. This block models a solar cell as a parallel combination of a current source, two exponential diodes and a parallel resistor, $\mathrm{Rp}$, that are connected with a series resistance Rs. Figure 2 shows the implementation of solar cell module in Simulink.

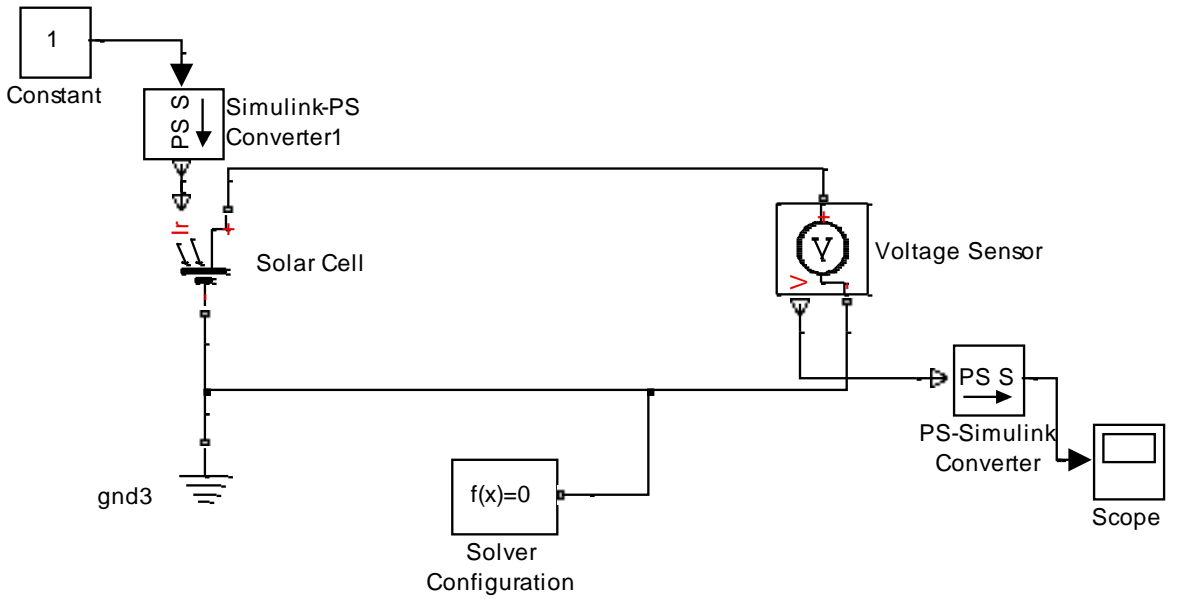

Figure 2. Solar Cell Module in Simulink

\section{Signal Conditioning Circuit}

The two small solar panels have a voltage rating of $600 \mathrm{mV}$, while the analog-to-digital converter (ADC) of the microcontroller will accept input voltage from 0 to $5 \mathrm{~V}$. A signal conditioning circuit is designed and built to interface between the small solar panels and the microcontroller. A non-inverting amplifier is built using an operational amplifier. The voltage gain of the non-inverting amplifier is designed to be 6. A Simulink simulation of the noninverting amplifier is shown in Figure 3. R1 is $2 \mathrm{k} \Omega$ and $\mathrm{R} 2$ is $10 \mathrm{k} \Omega$, which provides a gain of 6 .

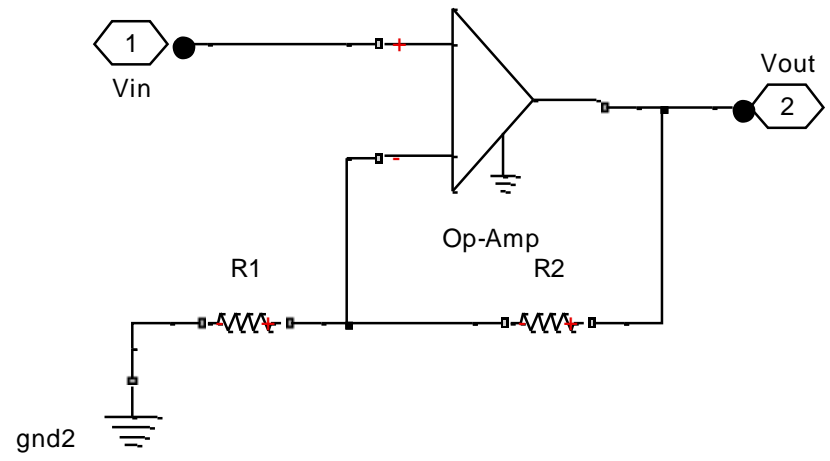

Figure 3. Simulink Simulation of Non-inverting Amplifier 


\section{Controller}

Figure 4 shows the control algorithm. The controller is implemented as an embedded Matlab function in the simulation. The first block is initialization that turns on power for all components. Next, the output voltage of the east and the west tracking cells are added to determine if it is daytime or night. If it is night, the program will repeat the loop. After sunrise, the program will jump out of the loop and proceed to daytime tracking loop. There are three situations based on the output voltage from east and west tracking cells. If the maximum rotation steps are reached, the controller will go to the night mode state. It will first rotate clockwise until the steps number is equal to 0 and then it starts to go into the sleeping state for certain delay period of time until sunrise. The embedded Matlab function allows convenient evaluation and modification of the control algorithm. In the prototype solar tracker system, the controller will be implemented using a microcontroller.

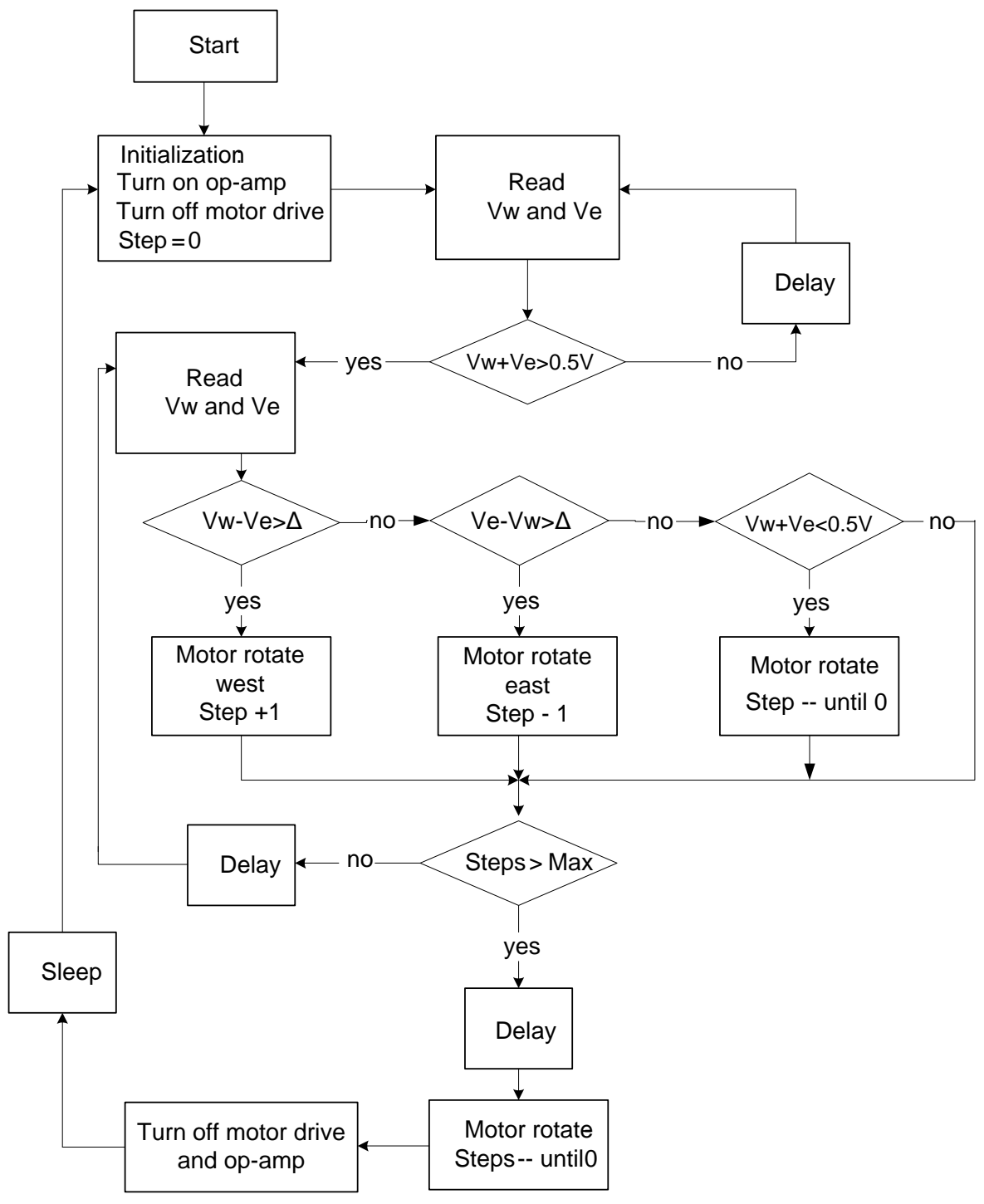

Figure 4. Control Algorithm 


\section{Motor and Drive}

Figure 5 shows the Simulink simulation of the stepper motor and drive. The motor driver has three input pins to control the motor move. One is step clock signal which is PWM signal used to control the motor rotate. The second input is direction pin used to control the direction of rotation by switch the logic between 1 and $0(0 / 5 \mathrm{~V})$. The third input is enable.

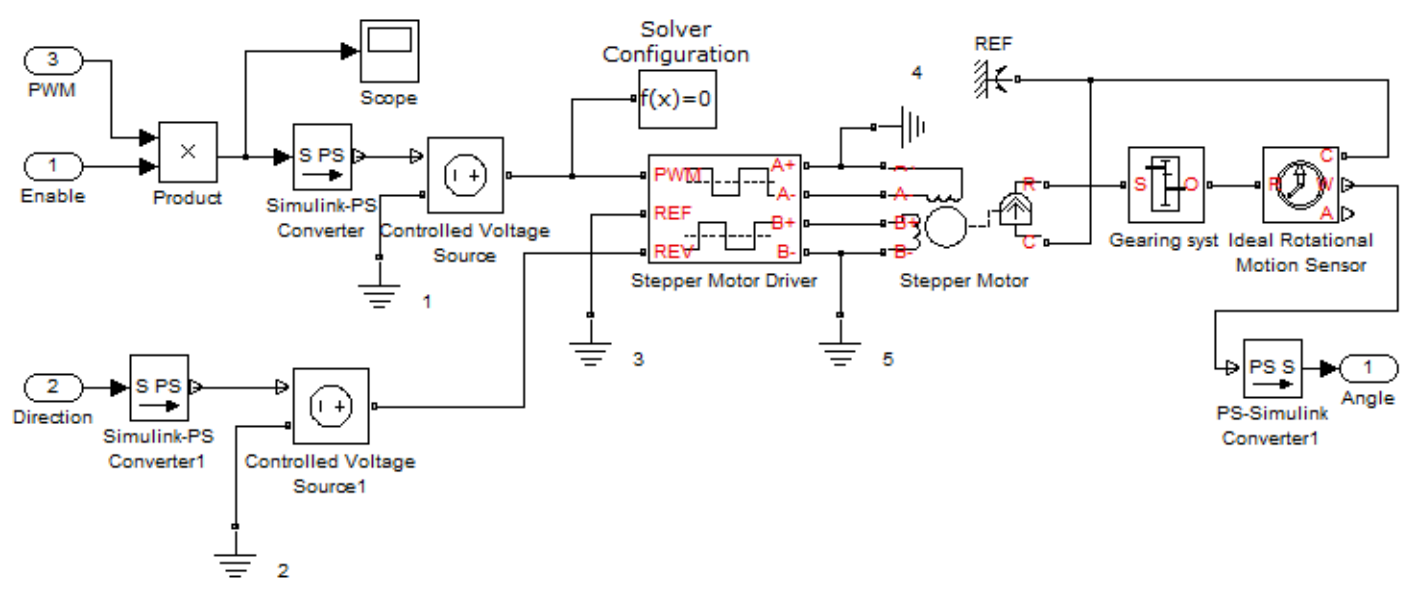

Figure 5. Simulation of Stepper Motor and Drive

The simulation of the tracking solar cells, signal conditioning, control and stepper motor is integrated, which completes the simulation for the sun tracking solar power system. The system is a feedback control system, as the angle of the main panel is sensed and sent back to the input of the system.

\section{Conclusion}

Students need knowledge of circuit theory, power electronics, microcontrollers, electric machines, and solar power in order to design and simulate the system. The broad scope of this project requires the students to investigate and address a wide range of complex issues and expose students to renewable energy technologies. The Matlab/Simulink simulation provided an excellent platform for the students to study the theory and explore different designs for the sun tracking solar power system. After testing and verification using the simulation, a prototype system will be built in the laboratory.

\section{Acknowledgement}

Partial support for this work was provided by the National Science Foundation's Transforming Undergraduate Education in Science, Technology, Engineering and Mathematics (TUES) program under Award 1140447. Any opinions, findings, and conclusions or recommendations expressed in this material are those of the authors and do not necessarily reflect the views of the National Science Foundation. 


\section{Bibliography}

1. C. Alexandru, C. Pozna, "Different tracking strategies for optimizing the energetic efficiency of a photovoltaic system", IEEE International Conference on Automation, Quality and Testing, Robotics, May 2008, Vol. 3, pp. 434 439

2. A. Yazidi, F. Betin, G. Notton, G. A. Capolino, "Low cost two-axis solar tracker with high precision positioning," First International symposium on Environment Identities in Mediterranean Area, July 2006, pp. 211 - 216

3. J. Beltran, etl. "Design, manufacturing and performance test of a solar tracker made by a embedded control," Proceedings of the Electronics, Robotics and Automotive Mechanics Conference, 2007, pp. 129-134

4. P. R. Mukund, Wind and Solar Power Systems, CRC Press, 1999

5. Ignacio Luque-Heredia, etl. "A sun tracking error monitor for photovoltaic concentrators," Electronics, Robotics and Automotive Mechanics Conference, Sept 2007, pp. 129 - 134

6. O. Oltu, etl. "Solar panel energetic efficiency optimization method, based on an specific detector and orientation microsystem," International Semiconductor Conference, Oct 2007, Vol. 1, pp. 127 - 130 